\title{
Aliou GAYE, Tourisme et patrimoine culturel : valorisations, enjeux et stratégies de développement local à l'île de Gorée et en pays Bassari (Sénégal)
}

Thèse de doctorat en tourisme et patrimoine, sous la direction de Dominique CHEVALIER (Université Claude Bernard-Lyon 1), soutenue publiquement le 29 juin 2020 à l'Université Lumière Lyon 2, 609 pages Tourism and cultural heritage: valuations, challenges and local development strategies on the island of Goree and in the Bassari country (Senegal)

\section{Aliou Gaye}

\section{OpenEdition}

\section{Journals}

Édition électronique

URL : http://journals.openedition.org/tourisme/2907

DOI : $10.4000 /$ tourisme.2907

ISSN : 2492-7503

Éditeur

Éditions touristiques européennes

Référence électronique

Aliou Gaye, "Aliou GAYE, Tourisme et patrimoine culturel : valorisations, enjeux et stratégies de développement local à lîle de Gorée et en pays Bassari (Sénégal) », Mondes du Tourisme [En ligne], 17| 2020, mis en ligne le 01 juin 2020, consulté le 20 décembre 2020. URL : http://

journals.openedition.org/tourisme/2907 ; DOI : https://doi.org/10.4000/tourisme.2907

Ce document a été généré automatiquement le 20 décembre 2020.

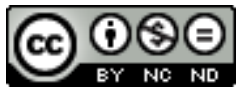

Mondes du tourisme est mis à disposition selon les termes de la licence Creative Commons Attribution - Pas d'Utilisation Commerciale - Pas de Modification 4.0 International. 


\section{Aliou GAYE, Tourisme et patrimoine culturel : valorisations, enjeux et stratégies de développement local à l'île de Gorée et en pays Bassari (Sénégal)}

Thèse de doctorat en tourisme et patrimoine, sous la direction de Dominique CHEVALIER (Université Claude Bernard-Lyon 1), soutenue publiquement le 29 juin 2020 à l'Université Lumière Lyon 2, 609 pages Tourism and cultural heritage: valuations, challenges and local development strategies on the island of Goree and in the Bassari country (Senegal)

\section{Aliou Gaye}

Conformément à une politique mondialisée de développement qui s'est récemment accélérée à travers différents processus, les termes de tourisme et de patrimoine sont marqués par une extension simultanée de leurs domaines d'activité et d'application. Le tourisme s'est révélé productif dès l'avènement du Grand Tour de l'aristocratie anglaise à la fin du XVII ${ }^{\mathrm{e}}$ siècle (Cousin et Réau, 2009), prémices à l'essor du tourisme de masse qui concerne aujourd'hui le tourisme culturel, l'écotourisme, le tourisme éthique, le tourisme responsable, le tourisme rural intégré, le tourisme de mémoire, le tourisme d'affaires, le tourisme de santé, le tourisme cynégétique, l'agrotourisme et le tourisme sexuel. Le patrimoine répond, quant à lui, à l'évolution des sociétés contemporaines et leur prise de conscience des héritages patrimoniaux, d'où l'émergence du patrimoine culturel, naturel, architectural, industriel, urbain, historique, religieux et subaquatique. Ces deux faits sociaux se déploient progressivement dans le champ scientifique et l'espace social, au point que certains chercheurs les qualifient de " couple de la mondialisation » (Lazzarotti, 2000) ou encore de phénomènes globaux, voire de réalités mondiales et de recherches savantes.

2 Dans cette logique, le postulat de notre travail de recherche repose sur l'idée selon laquelle le tourisme est à la fois un fait social, un phénomène spatial, un outil 
d'aménagement et une activité économique, dont les arrivées et les recettes touristiques ne cessent d'augmenter depuis la révolution industrielle et la démocratisation des congés payés (Violier, 2008). Quant au patrimoine culturel, il est avant tout un héritage social d'un peuple ou d'une communauté, nécessitant d'être sauvegardé, conservé et protégé en vue d'une transmission valorisée aux nouvelles et futures générations (Unesco, 2006). En effet, il constitue une dimension économique et politique, à travers laquelle les pratiques touristiques connaissent une double acception : les économies d'échelles et la dégradation du cadre de vie humain.

Cette thèse donne une lecture nuancée des interactions entre tourisme et patrimoine culturel. Elle se focalise plus spécifiquement sur la problématique des mémoires collectives de l'esclavage et des héritages coloniaux présents à l'île de Gorée, et sur la permanence et les mutations possibles des paysages culturels du pays Bassari. En étudiant les jeux d'acteurs, elle analyse les enjeux politiques, socioculturels, économiques et environnementaux. Elle appréhende aussi les imaginaires touristiques et les représentations sociales. L'objectif est de montrer comment le tourisme peut contribuer à la redynamisation de ces territoires, dans un contexte où les cultures locales sont menacées par les effets contrastés de la mondialisation. Si le tourisme est considéré comme l'un des facteurs clés de l'émergence économique de l'île de Gorée et du pays Bassari, il est aussi synonyme de danger pour leur héritage patrimonial. C'est précisément dans cette situation paradoxale que s'inscrit cette thèse visant à étudier les relations ambivalentes entre ces deux phénomènes sociaux - des liens qui peuvent être à la fois bénéfiques et nuisibles pour la population locale.

4 Notre choix d'étudier l'île de Gorée et le pays Bassari repose sur le fait que ces deux sites sont inscrits sur la Liste du patrimoine mondial de l'humanité. Gorée détient un riche patrimoine témoignant d'une expérience humaine unique dans les annales des sociétés africaines, européennes et américaines (Bocoum et Toulier, 2013). En effet, ce lieu de mémoire est, pour la conscience universelle, le symbole de l'esclavage, avec ses stigmates qui redessinent temporairement l'histoire des peuples africains dans le monde. Il est devenu à la fois un haut lieu mémoriel et touristique, où convergent une élite de personnalités mondialement connues et de nombreux touristes en quête de partage et d'expériences. Inscrite sur la Liste du patrimoine mondial de l'humanité depuis 1978, Gorée révèle une politique mémorielle plus ou moins ostentatoire ou voilée dans le processus de patrimonialisation de ses mémoires plurielles. Le pays Bassari bénéficie, quant à lui, de ressources patrimoniales exceptionnelles: manifestations culturelles, architectures, objets traditionnels et savoir-faire artisanal notamment (Girard, 1984), qui représentent d'importants potentiels de développement touristique. Classé au patrimoine mondial de l'Unesco depuis 2012, ce territoire est aujourd'hui à une étape charnière de son histoire. Il attire de nombreux touristes et populations étrangères, ce qui pourrait conduire à déstabiliser les modes de vie traditionnels des habitants locaux. 


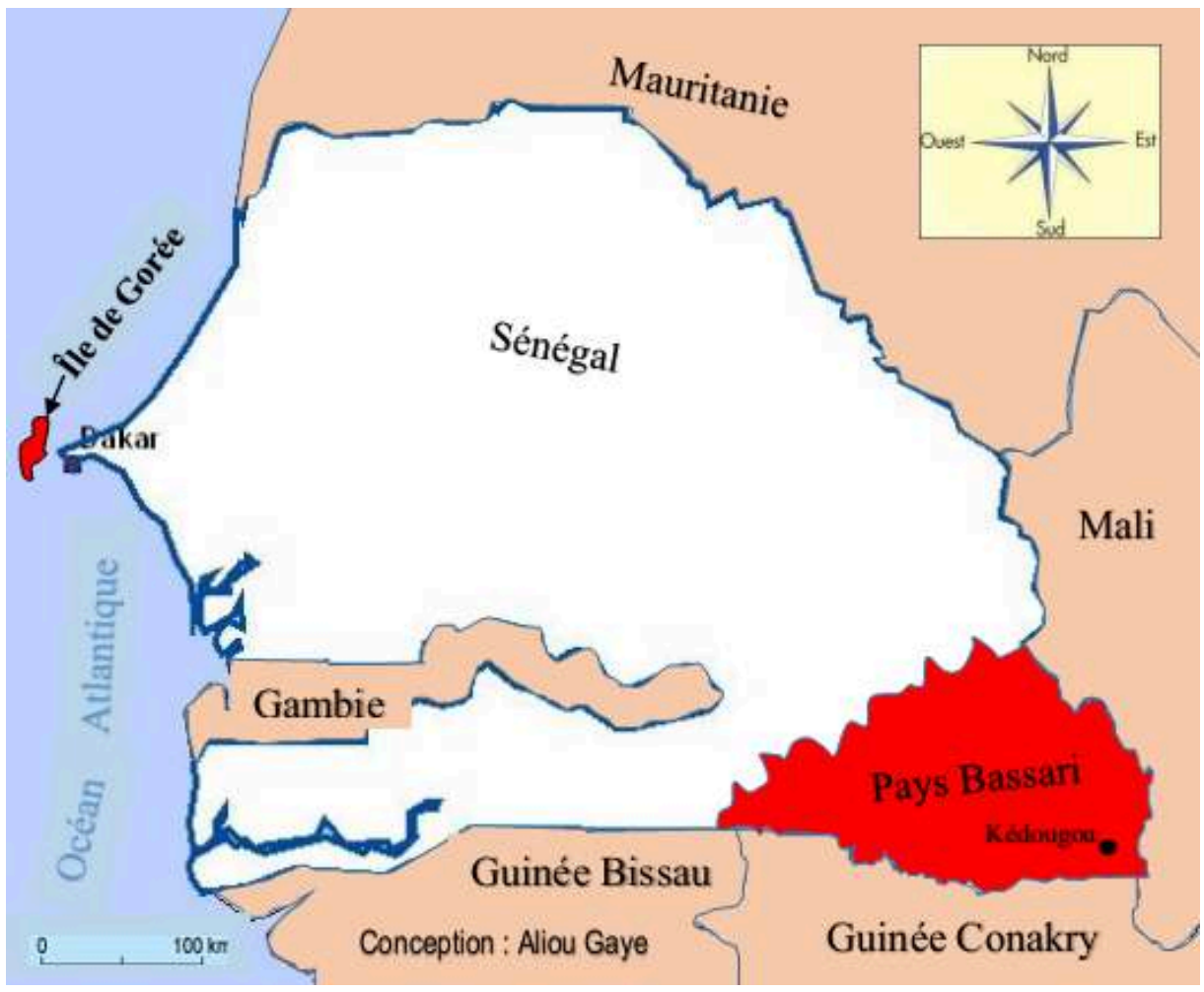

Source : auteur

5 Du point de vue méthodologique, l'observation participante nous a semblé la plus adéquate pour comprendre les réalités du terrain. Nous avons en outre choisi d'aborder différents acteurs par le biais de questionnaires et d'entretiens semi-directifs. À Gorée, nous avons effectué un séjour de trois mois, durant lesquels nous avons mené un état des lieux du patrimoine. En pays Bassari, notre étude de terrain a duré cinq mois dans les villages bassari, bédik et peuls. Dans cette démarche, 70 questionnaires ont été administrés auprès des populations locales, d'artistes, de touristes, d'élèves et d'étudiants, et des entretiens menés avec des acteurs publics et privés, sur la base d'un guide d'entretien ouvert préétabli (167 questions). Parmi les personnes ressources interrogées, figurent des représentants du ministère du Tourisme et de la direction du Patrimoine, mais également des gestionnaires de sites patrimoniaux, des élus locaux, des chefs coutumiers, des chefs de village, des représentants de l'Unesco à Dakar et des partenaires d'appui au développement local. Afin de mieux appréhender ces différents éléments, nous avons choisi de loger chez l'habitant, puisqu'il s'agissait bien d'étudier les pratiques culturelles et touristiques, pour lesquelles la population locale représente le noyau essentiel de tout projet de développement.

Notre approche s'articule autour de trois parties, composées chacune de deux chapitres, questionnant les relations complexes entre tourisme et patrimoine culturel à Gorée et en pays Bassari, englobant des enjeux multiples. La première est consacrée aux problématiques d'un patrimoine culturel en émergence grâce au tourisme. Le premier chapitre porte plus spécifiquement sur les «regards croisés entre destination touristique et tensions mémorielles à l'île de Gorée ", où on assiste à une mise en valeur des mémoires collectives de l'esclavage et des héritages coloniaux dans un dilemme politique. Ce chapitre étudie cette «île-historique» à la fois en tant que haut lieu 
mémoriel, haut lieu touristique, espace culturel et artistique, et questionne les discours, les récits et les mises en scène de ce lieu patrimonialisé. Le deuxième chapitre examine «les paysages culturels appréhendés comme patrimoines vivants en pays Bassari ", dont les ressources constituent des potentiels de développement touristique.

La deuxième partie explore les relations ambivalentes entre tourisme et patrimoine culturel. Le troisième chapitre porte sur «La fabrique du patrimoine culturel dans l'espace touristique ». Il étudie conjointement le tourisme et le patrimoine dans leurs interactions, en interne et en externe, en vue d'examiner leurs traits communs, leurs divergences et leurs apports. En tant que tel, il démontre que l'événementiel est un outil de communication, de valorisation et de promotion des ressources culturelles. Le quatrième chapitre évoque "La dimension matérielle et immatérielle des biens culturels et le monde des esprits en pays Bassari». Il analyse les ressources patrimoniales de ce territoire, s'inscrivant à la fois dans les ancrages paysagers et identitaires et se situant à la croisée des chemins entre tradition et modernité.

Enfin, la troisième partie aborde les risques et les ressources d'une mise en valeur touristique des héritages patrimoniaux. Le cinquième chapitre, intitulé «Processus de patrimonialisation et logiques de développement touristique ", examine la mise en patrimoine et la mise en tourisme des biens patrimoniaux. Il explore les dynamiques patrimoniales des mémoires collectives et des paysages culturels, suscitant parfois des revendications identitaires et territoriales. Le sixième chapitre, intitulé "Analyses touristiques des ressources patrimoniales: jeux d'acteurs et appropriation de l'espace ", interroge l'état de conservation de ce patrimoine. Il établit des pistes de réflexion sur la gestion touristique des biens patrimoniaux à l'échelle locale, nationale et internationale afin de trouver des solutions durables. En tant que tel, il définit les stratégies de communication que les professionnels du tourisme et les experts du patrimoine mettent en place pour protéger, valoriser et promouvoir leurs héritages patrimoniaux à des fins touristiques.

L'analyse des interactions entre tourisme et patrimoine nous permet finalement de mesurer les impacts des pratiques touristiques sur le développement local à l'île de Gorée et en pays Bassari. Le tourisme crée des emplois et génère des retombées économiques. Ainsi, à Gorée, il représente la première activité économique, permettant de faire vivre les habitants locaux. En pays Bassari, il a favorisé l'aménagement de l'espace par la construction de routes goudronnées, d'hôtels, de dispensaires, d'écoles et de centres multimédias. Mais, si le tourisme est considéré comme l'un des facteurs clés de l'émergence économique de ces territoires, il représente aussi une menace pour leurs patrimoines. Il bouleverse les modes de vie des populations locales, qui peuvent se trouver affectés par différentes dérives: prostitution, mendicité, folklorisation des pratiques culturelles, coût élevé du loyer, cherté des produits locaux, litiges fonciers... Se confirme ainsi l'hypothèse selon laquelle les populations locales doivent être sensibilisées davantage aux enjeux du tourisme: il s'agit de les impliquer dans les projets de patrimoine et de tourisme afin de mieux assurer, de façon durable, la gestion touristique de leurs ressources patrimoniales. Les touristes doivent également être sensibilisés au respect des modes de vie des habitants locaux et se conformer à la législation du pays visité. Dans cette prise de conscience collective, une pédagogie patrimoniale s'impose auprès des jeunes scolaires, afin d'éveiller leur esprit à la protection du patrimoine. 


\section{BIBLIOGRAPHIE}

Hamady воcoum et Bernard TOULIER, « La fabrique du patrimoine : l'exemple de Gorée (Sénégal) », IN SITU Revue des patrimoines, $\mathrm{n}^{\circ} 20,2013$.

Saskia CoUSIN et Bertrand RÉAU, Sociologie du tourisme, La Découverte, 2009.

Jean GIRARD, Les Bassari du Sénégal, Fils du Caméléon. Dynamique d'une culture troglodytique, L'Harmattan, 1984.

Olivier LAZZAROTTI, « Patrimoine et tourisme : un couple de la mondialisation », Mappemonde, vol. $57, \mathrm{n}^{\circ} 1,2000$.

Philippe VIOLIER, Tourisme et développement local, Belin, 2008.

UNESCO, Patrimoine culturel et développement local, CRATerre-ENSAG/Convention France-UNESCO, 2006.

\section{AUTEUR}

\section{ALIOU GAYE}

Aliou.Gaye[at]univ-lyon2.fr 